\title{
PROBlematiCHE Di TRADUZIONE LETTERARIA DALL'ITALIANO IN ARABO IN UN MODELLO DIDATTICO APPLICATO
}

\author{
Samah Mobammed Ibrabim $A b d 0^{1}$
}

\section{INTRODUZIONE}

L'atto del tradurre presuppone una vera e propria sfida stimolante che mette in gioco tutte le abilità sia interpretative sia creative del traduttore. L'obiettivo dell'articolo è quello di evidenziare le problematiche di natura semantico-lessicale nella traduzione letteraria compiuta da 7 gruppi di studenti egiziani e arabofoni al quarto anno del corso di Laurea in Lingua e letteratura italiana all'anno accademico 2019/2020, 49 studenti in totale.

Presso l'ateneo di Ain Shams, gli studenti d'Italianistica fanno un corso di quattro anni accademici per raggiungere alla fine il livello di C2. Al termine dello studio si deve presentare una tesi di laurea in grammatica, letteratura o traduzione. Per poter accedere a questo corso, è necessario aver raggiunto il livello B2, in modo da poter capire le caratteristiche della lingua italiana, comprendere quali sono le principali differenze fra l'arabo, la madre lingua, e l'italiano, seconda lingua (SL), ed elaborare una tesi di laurea con un approccio critico e scientifico.

Nel corpus sono stati inclusi 7 testi di opere letterarie di varie tipologie, racconti, romanzi, fumetti, favole e fiabe; ogni tipologia presenta, almeno in parte, delle problematiche diverse. Per l'analisi abbiamo avuto la traduzione, intere o in parte, di:

1. Gianni Rodari, Favole al telefono, Edizione Einaudi ragazzi, Torino, 1995: (Rodari, FT)

2. Gianni Rodari, Le avventure di Cipollino, Editore Einaudi Ragazzi, Torino, 2018: (Rodari, AC)

3. Grazia Deledda, Racconti sardi, Editore Giuseppe Dessi, Sassari, 1894: (Deledda, RS)

4. Italo Calvino, Fiabe Italiane, Oscar Mondadori, Milano, 2015: (Calvino, FI)

5. Italo Svevo, I racconti, e-book, www.liberliber.it, Edizione elettronica, 2010: (Svevo, R)

6. Luigi Pirandello, Novelle per un anno, e-book, www.liberliber.it, II edizione elettronica, 2013: (Pirandello, NA)

7. Walter Venturi, Il grande Belzoni, Romanzi a fumetti Bonelli, Sergio Bonelli Editore, Milano, 2013: (Venturi, GB)

Lo strumento principe per la traduzione è il dizionario; indispensabili sono un dizionario bilingue italiano-arabo, come $A l \mathrm{Tellis} \mathrm{L}^{2}$, e monolingue italiano - italiano, come il Grande Dizionario Garzanti ${ }^{3}$, disponibile quasi per tutti gli studenti sia in formato cartaceo che digitale, e un dizionario arabo-arabo come $A l-M a a n y^{4}$, insieme a un'enciclopedia come Treccani ${ }^{5}$.

\footnotetext{
${ }^{1}$ Università di Ain Shams. Facoltà di Al-alsun.

${ }^{2}$ Kalifa M. Tellisi (1986).

${ }^{3}$ Grande Dizionario Garzanti di Italiano (2006).

${ }^{4}$ https://www.almaany.com/.

5 https://www.treccani.it/.
} 
L'approccio è improntato sia all'autonomia sia alla collaborazione: in autonomia gli studenti traducono cercando delle soluzione ai problemi; in classe poi discutono insieme al docente le sfide e le soluzioni possibili; qui il docente mette a disposizione le sue competenze traduttologiche. Le dinamiche comunicative e le discussioni rendono il corso un vero e proprio laboratorio di traduzione. Seguendo i suggerimenti del docente ${ }^{6}$ gli studenti devono effettuare l'autocorrezione degli errori e quelli più ricorrenti vengono trattati nelle lezioni. Con l'analisi delle interferenze linguistiche e delle problematiche è possibile preparare le lezioni in cui il docente spiega le strategie di traduzione in modo da offrire agli studenti un sostegno per affrontare le difficoltà e renderli consapevoli del modo di autocorreggersi.

Mi sono focalizzata sull'analisi delle difficoltà semantico-lessicali, partendo sempre dagli esempi presi dal nostro corpus; non verranno trascritte sempre frasi intere ma il contesto sufficiente per capire l'errore. In particolare, sono stati analizzati: le espressioni idiomatiche, la polisemia, i giochi di parole, gli antroponimi e le onomatopee.

\section{LE ESPRESSIONI IDIOMATICHE}

Tra gli errori lessicali, numerosissimi sono quelli appartenenti alle espressioni che rappresentano un ostacolo per gli stranieri traduttori. Le espressioni idiomatiche, quelle sequenze fisse di significato opaco, vengono così definite da Federica Casadei:

[sono] espressioni convenzionali di una lingua caratterizzate dall'abbinare un significante fisso (poco o affatto modificabile) a un significato non composizionale (cioè che, a differenza del significato letterale o composizionale, non è ricavabile dai significati dei componenti dell'espressione). (Casadei, 1995: 335)

Indipendentemente dal significato letterale, l'espressione non può essere pienamente compresa dai significati separati dei suoi singoli componenti, ma deve invece essere appresa come unità di significato. A livello sintattico e semantico, Langlotz (2006: 3-4) indica quattro caratteristiche delle espressioni idiomatiche; institutionalisation (il grado di convenzionalità e familiarità di un'espressione idiomatica appartenente a una comunità di parlanti), compositeness (in quanto sono strutture linguistiche costituite da due o più elementi lessicali), frozeness (come risultato di un processo convenzionale delle unità lessicali, considerate non più nella loro singolarità, ma nel loro insieme) e noncompositionality (una lettura di tipo composizionale non è sufficiente per cogliere il senso idiomatico dell'espressione. Si parla allora di significato non- composizionale). Di fronte a questo fenomeno linguistico molto vasto e disomogeneo le difficoltà consistono sia nell'ambiguità tra senso letterale e senso figurato, sia dall'opacità del messaggio e nella maggior parte dei casi ne nasce una traduzione letterale dell'espressione.

A volte le espressioni sono interamente o parzialmente «motivate» (Alliaj, 2018/2019: 24) cioè comprensibili dai significati letterali delle singole parole costituenti. Queste sono espressioni non idiomatiche, poiché gli elementi che le costituiscono non presentano un significato figurato. Le espressioni idiomatiche sono quelle che implicano delle figure di significato, quali metafore, metonimie, iperboli o altre figure retoriche, e vengono definite «non motivate» (ibid.) perché non si può dedurre il loro significato dagli elementi

${ }^{6}$ Il docente segna le interferenze linguistiche poi le classifica, fornendo a ogni singolo studente di un breve feedback. Se lo studente non è capace di correggere un errore, gli viene fornita la risposta corretta prima dagli altri studenti dello stesso gruppo, o, dopo, dal docente. 
costituenti. Qui entra in gioco la dicotomia trasparenza / opacità semantica che riguarda il grado di difficoltà con cui un traduttore riesce a capire e tradurre un'espressione. Maggiore è la differenza tra il significato letterale e il significato idiomatico, più grande è l'equivoco (Langlotz, 2006: 4). Sul binomio trasparenza / opacità, si è soffermata anche Casadei (1996: 391) mostrando come la relazione tra il significato idiomatico e quello letterale non è necessariamente opaca; se un traduttore posto davanti a un'espressione idiomatica riesce a capirne il significato grazie alla comprensione dei singoli termini, siamo davanti a un'espressione trasparente.

Proprio a causa delle caratteristiche sopra menzionate le espressioni idiomatiche sono fonte di molti errori da parte degli studenti, ma anche di quelli che hanno un'alta competenza linguistica: la conoscenza delle singole parole non ci permette di accedere al messaggio. Si è deciso di partire dalla ricerca di un equivalente nella lingua d'arrivo, o dalla modulazione dell'espressione per evitare una traduzione letterale:

Tabella 1. Espressioni idiomatiche

1. Allora il Re mandò fuori il bando che tutte le ragazze da marito venissero al giardino, pena la testa

(Calvino, FI: 61)

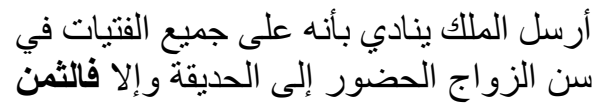

حياتهن الزون

2. Ma il vagabondo non fu lesto a prenderla al volo

(Calvino, FI: 94)

3. Eccomi, senza arte né parte a girovagare per i circhi e le piazze

(Venturi, GB: 20)

4. Voglio fare otto giorni di corte bandita

(Calvino, FI: 99)

5. Gli aveva fatto sparire sotto il naso tutti i prigionieri

(Rodari, AC: 140)

6. Meglio godersi questa rilassante crociera... presto dovremo usare l'olio di gomito

(Venturi, GB: 110)

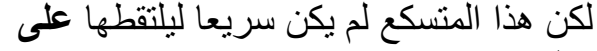

الطاير.

ولكن ها أنا ذا، بلا شغلة ولا مشغلة، اتنقل بين سيرك وأخر و عبر الميادين.

أود أن أقيم وليمة لثمانية أيام

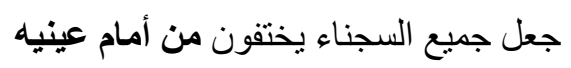

الأفضل أن نستمتع بهذه الرحلة النيلية، حيث ينتظرنا عرق الجبين

Come gli esempi mostrano, gli studenti hanno cercato d'individuare il significato delle espressioni nella lingua di partenza e di trovarne gli equivalenti nella lingua d'arrivo. La locuzione "pena la testa" equivale in arabo alla locuzione "الثمن حياتهن" ("il prezzo è la loro vita"), l'espressione "corte bandita" equivale a "وليمة" ("grande banchetto)". Nella locuzione "prendere a volo" si opta per un'espressione colloquiale " "على الطاير" ("prima che rimbalzi a terra"), una traduzione che non compensa il registro linguistico del testo di partenza, proprio come "senza arte né parte" che viene tradotto in modo colloquiale " "شغلة ولا مشغلة" (“senza nessuna occupazione”).

Gli studenti adottano un approccio source-oriented, una traduzione orientata il più possibile verso il testo di partenza, per raggiungere una versione fedele all'originale. Quest'approccio dipende in particolar modo dal concetto di equivalenza, introdotto da Eugene Nida secondo cui non esiste un'assoluta corrispondenza fra il testo di arrivo e il testo di partenza, ma può esserci un'equivalenza dinamica o formale; l'equivalenza formale concentra l'attenzione sia sulla forma che sul contenuto del messaggio puntando 
a rendere il più chiaro possibile il contesto di partenza al ricevente, mentre l'equivalenza dinamica ha l'obiettivo di suscitare l'effetto equivalente, per cui la relazione tra il ricevente e il messaggio in lingua di arrivo deve essere uguale alla relazione tra il ricevente e il messaggio in lingua di partenza (Nida, 1964: 159).

Prendendo spunto dalle teorie sull'equivalenza, è compito degli studenti trovare strutture ed espressioni equivalenti a quelle del testo originale, come nella traduzione della maggior parte delle locuzioni riguardanti il campo semantico del corpo, quelle che provengono dall'osservazione visiva degli uomini; "sotto il naso" = "أمام عينيه" con il significato di "davanti agli occhi", e "l'olio di gomito" = "عرق الجبين" cioè "il sudore della fronte" dove però "il gomito" viene sostituito da "la fronte" e "l'olio" da "il sudore", una traduzione che mantiene il contenuto semantico e figurato dell'espressione originale.

Quando intervengono procedimenti retorici nella costruzione delle espressioni idiomatiche, la traduzione fedele del testo risulta più ardua. Le teorie formulate con l'approccio source-oriented vengono chiamate prescrittive di cui è un esempio lo studio di Klingberg (1986) secondo il quale la traduzione deve rimanere fedele all'originale e il testo di partenza deve essere manipolato il minimo possibile. Per dimostrare quanto detto, riportiamo di seguito alcuni esempi tratti dalle traduzioni degli studenti:

Tabella 2. Espressioni idiomatiche

\section{Qui il diavolo ci ba messo la coda}

$$
\text { (Rodari, AC: 140) }
$$

2. Ne combinava di tutti $i$ colori

$$
\text { (Rodari, FT, 59) }
$$

3. La fanciulla esitava, le guance in fiamme

$$
\text { (Svevo, R: 39) }
$$

\section{Non dispone del becco di un quattrino!}

(Svevo, R: 39)

\section{Non sanno mai dove andare a battere il capo}

(Rodari, AC: 50)
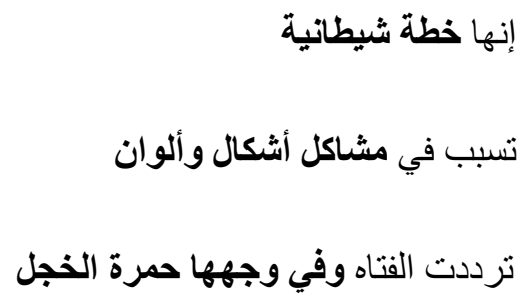

لا يملك فلسا واحد!!

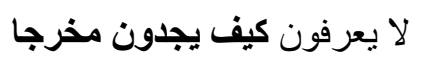

In queste traduzioni il significato si crea nell'adeguamento alle strutture linguistiche e culturali della lingua di arrivo, come nelle espressioni "Qui il diavolo ci ha messo la coda" tradotta "إنها خطة شيطانية" ("un piano diabolico”) che mantiene la metafora del testo

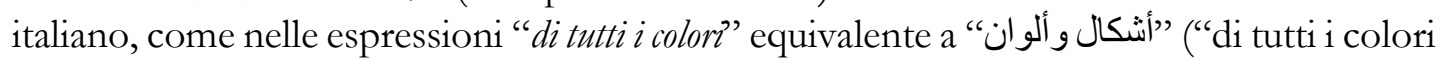
e tutte le forme"), "guance in fiamme" corrispondente a "وفي وجها حمرة الخجل" "con le guance rosse di pudore"). Tuttavia non sempre all'espressione idiomatica italiana ci si riesce a trovare un'espressione equivalente araba. Lo studente potrebbe conoscere il significato dell'espressione e optare per una generalizzazione o una parafrasi, come nell'espressione "becco di un quattrino" tradotta "فلسا واحدا" ("non ha neanche un quattrino"), e l'espressione idiomatica "andare a battere il capo" tradotta " يجدون مخرجاو "'senza via d'uscita"); sono due esempi in cui le traduzioni conservano il contenuto semantico ma non quello figurato delle espressioni italiane.

Non mancano i casi di una sostituzione analitica delle espressioni: 
(C) Italiano LinguaDue 2. 2021. Samah Mohammed Ibrahim Abdo, Problematiche di traduzione letteraria dall'italiano in arabo in un modello didattico applicato

Tabella 3. Espressioni idiomatiche

1. Per riscaldarsi, lanciava in croce le braccia

(Svevo, R: 167)

2. Non mi faceva grazia nelle sue lettere di nessuno dei tanti palpiti che quella bonifica gli era costata.

(Pirandello, NA: 1025)

3. Dunque in testa.. Bell'e andato!

(Pirandello, NA: 2318)

4. Aveva messo radice in quel paradiso

ليحصل على بعض الدف،، ربع يديه

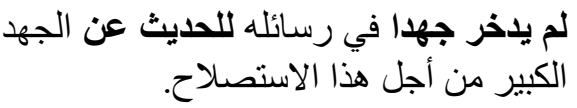

استهدف رأسه.. لقد انتهى أمره!

كان قد استقر في تلك الجنة

(Svevo, R: 5)

5. Se non rispondi, fatti la croce!

إن لم تجب، فاستعد للموت!

(Pirandello, NA: 2317)

L'espressione "lanciava in croce le braccia" viene tradotta con la modulazione lessicalizzata "ربع يديه" ("ha incrociato le braccia"), "non faceva grazia ... di nessuno dei tanti palpiti” tradotta " "لم يدخر جها في في رسائله للحديث عن" "faceva di tutto per parlarne nelle sue lettere"), "Bell'e andato" tradotta "لقد انتهى أمره" ("è già finito"), "Aveva messo radice" tradotta semplicemente

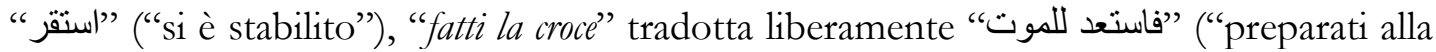
morte"). Non è sempre facile trovare termini arabi che rendano con la stessa sinteticità e pregnanza gli stessi concetti, soprattutto nelle espressioni che esprimono gestualità, quindi si opta per una traduzione che perde la struttura della similitudine / metafora, pur conservando il contenuto semantico.

Le espressioni idiomatiche presenti nel nostro corpus, infatti, possono essere motivo di esitazione; il non avere un equivalente arabo può portare a una traduzione letterale. A volte gli studenti non riescono a rintracciare le espressioni idiomatiche nei dizionari a disposizione e cadono in una traduzione letterale infedele che ha delle conseguenze sulla comprensione da parte del lettore, sullo stile e sull'intenzione comunicativa dell'autore:

Tabella 4. Espressioni idiomatiche

1. Il naso guardava fisso davanti a sé, come un vecchio lupo di lago

ظل الأنف ينظر في ثبات للأمام كما لو كان

(Rodari, FT, 60)

2. Intorno all' ascensore si allargava ormai ai quattro venti lo spazio interpalentario

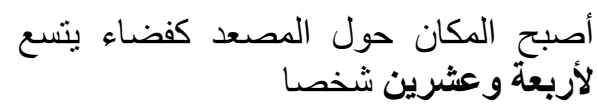

(Rodari, FT, 114)

3. E dunque non ci si vedeva neanche a tirar moccoli.

(Pirandello, NA: 1028)

4. Appena messo il naso fuori del nido,...

لم يكن يمكن للمرء حتى ينظف أنفه.

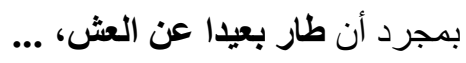

(Svevo, R: 190)

5. Occhio con le donne arabe!

(Venturi, GB: 105)

6. Trovai mia moglie con un diavolo per capello

(Pirandello, NA: 354) 
"بحار قديم" Negli esempi, “lupo di lago" viene tradotto letteralmente "ذئب بحيرة" invece di" cioè "marinaio esperto", l'espressione numerica "ai quattro venti" subisce anch'essa una

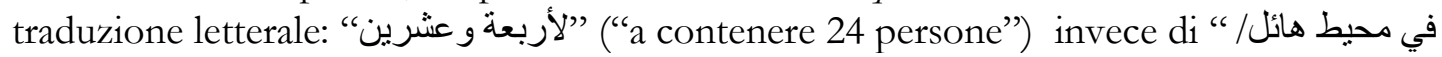

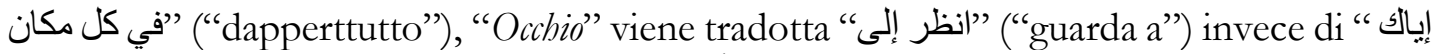

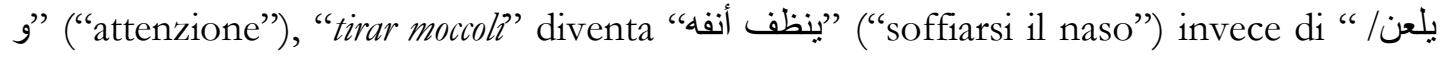
"' "bestemmiare, imprecare") e l'espressione "con un diavolo per capello" viene tradotta

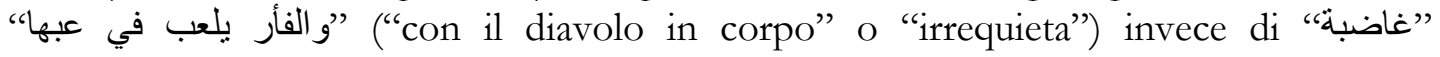
("arrabbiata"). A volte i dizionari monolingue presentano delle lacune e quelli bilingui si limitano a dare solo la traduzione letterale dell'espressione o la spiegazione del suo significato, senza indicarne l'equivalente (cfr. Ulrych, 1992: 249), anzi nella maggior parte dei dizionari italiano-arabo, non vengono raccolte le espressoini. Quindi, considerata la mancanza di repertori bilingui aggiornati con le locuzioni standarizzate e di uso corrente di espressioni gergali, gli studenti cadono in una traduzione letterale.

L'analisi degli esempi ha dimostrato che la traduzione delle espressioni idiomatiche rappresentano una sfida particolare; le maggiori difficoltà si presentano nel campo della semantica, poichè qui la "perversione" del lessico tocca il suo apice. Il processo traduttivo deve quindi tenere conto della natura formale delle espressioni, che sono enunciate con almeno due sensi differenti: uno letterale e l'altro figurato.

\section{LA POLISEMIA}

Si dice polisemica una parola che ha molteplici significati collegati sia etimologicamente sia semanticamente, trovandosi sotto la stessa voce nel dizionario ( $\mathrm{cfr}$. Dardano, 2017: 163). Le parole polisemiche rappresentano una grande sfida per la traduzione; capire il contesto diventa indispensabile per poter tradurre una parola / espressione polisemica, altrimenti la traduzione risulta errata (cfr. Slapek, 2016: 43); ne è un esempio la tabella seguente che mostra le difficoltà che gli studenti affrontano trattando le parole polisemiche presenti nel nostro variegato corpus:

Tabella 5. La polisemia

1. La piccola figurina esitò, sforzò, si spostò per alžarsi

(Svevo, R: 41)

2. Il piccolo busto slacciato sulla camicia bianchissima ... (Deledda, RS: 27)

3. ... scolaro del liceo e del ginnasio

(Svevo, R:16)

4. Solo quando fu a una certa distanza,..

(Rodari, FT: 114)

5. Le hanno rubato il naso e le hanno lasciato la zucca? (Rodari, FT: 59)

6. Una ragazza preparava il pranzo accanto al focoalre e al suo costume la riconobbi per fonnese.

(Deledda, RS: 26)
تحرك التثثال الصغير متونراً، مجبراً، محاو لاً

النهوض

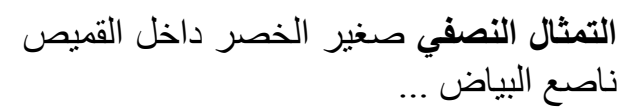

- م تلميذ بالمدرسة العليا وصالة الالعاب

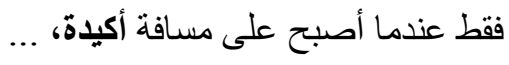

هل سرقوا أنفه وتركو ا له اليقطينة؟

كانت هنالك فتاة تحضر الغداء بجوار الموقد، حسب تقاليدها عرفت أنها من فونيز. 
Il termine polisemico "figurina", dim. di figura, significa "immagine disegnata, dipinta o scolpita", ma tra gli altri significati abbiamo: "il modo in cui qualcuno o qualcosa si mostra o appare". La parola ha messo alla prova gli studenti e subisce una traduzione letterale "تمثنال صغير" ("statuetta"), mentre il significato che l'autore intende è il secondo che dà un giudizio negativo alla ragazza che esita a muoversi. Abbastanza simile è la parola "busto" usata per dire "la parte del corpo umano compresa tra il collo e i fianchi", viene tradotta "تمثال نصفي", cioè "scultura che rappresenta una figura umana dalla testa al petto".

Allo stesso modo le polisemie dei termine "ginnasio" e "certa" vengono tradotte in modo inadatto al contesto come "(المدرسة الثانوية" "("palestra”) scartando",

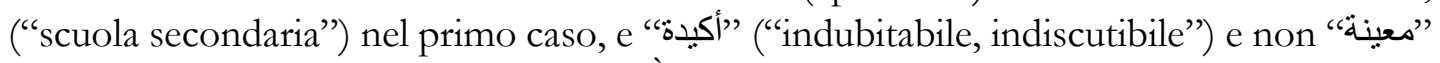
("specifico, determinato") nel secondo. È indispensabile effettuare un'analisi del contesto cercando elementi che ci possano indicare il senso della parola polisemica.

A volte, la questione si fa complicata, come nel termine "qucca" tradotto “"يقطينة" (inteso come "frutto commestibile di una pianta dallo stesso nome") mentre nel testo indica scherzosamente "testa", si dice: "battere la zucca contro uno spigolo e zucca pelata". Sembra che il testo ricco di fantasia li abbia ingannati. Un altro doppio senso è quello della parola "costume" utilizzata nel senso di "abito, indumento che si indossa in particolari occasioni”, ma tradotta in modo inappropriato come "تقاليد" ("complesso di usanze, abitudini"): gli studenti si confondono perché prima si parlava dell'ambiente fonnese, quindi non sono riusciti a individuare il significato più vicino alle intenzioni dell'autore.

Oltre ad optare per una parola inappropriata nella traduzione di un termine polisemico, un altro grave errore è quello di adattare la parola sbagliata al contesto. Gli esempi sono numerosi:

Tabella 6. La polisemia

1. Il principe non accennava a guarire.

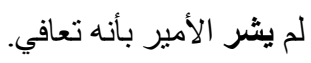

(Calvino, FI: 73)

2. È così che mi vuoi, Giovanni? Ai piedi, in paziente attesa?

(Venturi, GB: 127)

3. Essendosi recata sola, all'alba, giù nelle macchie del Conventino ...

(Pirandello, NA: 1776)

4. Mi ricambiò lo sguardo con aria di trionfo

(Pirandello, NA: 971)
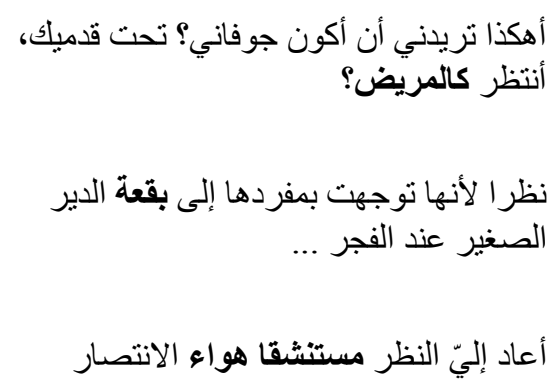

5. Permettete? Chiese con aria umile

(Pirandello, NA: 1060)

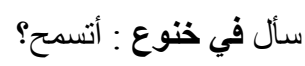

Il termine “accennare" viene usato nel testo senso di "يوحي بـ" ("manifestare l'intenzione"), mentre gli studenti lo traducono "يشر", ("indicare"), mentre il termine "paziente" invece di essere tradotto come aggettivo "بصبر", gli studenti optano per il sostantivo "مريضة" ("chi è affetto da una malattia"). Nei due esempi i termini scelti come traduzione vengono adattati al contesto: nel primo esempio " لميشر بالتعافي" viene adattata in questo modo: "Non fa cenni a quelli intorno che è stato guarito", e nel secondo dipendono dalla somiglianza "في صبر كالمريض" ("aspetta come un malato"). Un'altra

${ }^{7}$ Per gli approfondimenti, si consutla il Grande Diæ̧ionario Garzanti di Italiano (2006). 
parola di valore polisemico è "macchie" che viene tradotta letteralmente "بقعة"; mentre "macchia" significa fra l'altro "area di colore diverso dal resto di una superficie", in arabo "بقعة" ha come senso retorico "zona, quartiere", quindi l'errore è dovuto all'equivoco tra la lingua madre e l'italiano, mentre qui significa semplicemente "غابة" cioè "boscaglia fitta". Anche la parola "aria" nelle due espressioni polisemiche "con aria" crea ambiguità; essa assume il significato di "atteggiamento o espressione". Si dice: "avere un'aria stanca". في " Gli studenti scelgono una traduzione adatta al contesto solo nel secondo esempio " "خنوع", mentre nel primo la traducono "'respirando l'aria di trionfo") sforzando la parola scelta al contesto. Questo tipo di errore è dovuto in gran parte a una traduzione affrettata in cui lo studente sceglie un termine in base ad un significato che conosce già, senza consultare il dizionario per altri significati più convenienti.

Le parole polisemiche costituiscono una delle difficoltà più frequenti nella traduzione in lingue straniere perché a ogni parola possano corrispondere vari significati. Quando si devono trattare parole polisemiche, di solito sorgono traduzioni errate e per orientarci si deve cercare spunti nel contesto per scegliere i significati convenzionali appropriati al contesto; non basta consultare il dizionario perché a volte i dizionari registrano i diversi significati senza sottolineare i contesti in cui vengono usati.

\title{
4. I GIOCHI DI PAROLE
}

Nel gioco di parole vengono messe in relazione le strutture linguistiche con diversi significati sulla base della loro similarità formale creando una specie di ambiguità. $\mathrm{Ne} L a$ nuova grammatica della lingua italiana il gioco di parole viene definito «figura retorica basata sull'equivoco fonico (v. paronomasia) o semantico (v. anfibologia); s'incontra spesso nel linguaggio pubblicitario» (Dardano, Trifone, 1997: 643). Tale equivoco può essere dovuto alla sostituzione di una parola con un'altra o un'allusione per mezzo del doppio senso della parola usata. Non sempre è facile, dunque, tracciare un confine netto tra giochi di parole e alcune figure retoriche, dato che l'uso creativo della lingua si presta per sua natura a sfuggire a qualsiasi tentativo di rigida classificazione (cfr. Redfern, 1984: 9-21). Dirk Delabastita, ricercatore belga che ha svolto studi approfonditi sulla traduzione dei giochi di parole, propone la definizione seguente:

\begin{abstract}
Wordplay is the general name for the various textual phenomena in which structural features of the language(s) are exploited in order to bring about a communicatively significant confrontation of two (or more) linguistic structures with more or less similar forms and more or less different meanings. (Delabastita, 1996: 128)
\end{abstract}

Nel nostro corpus, l'opera più ricca di giochi di parole è Favole al telefono che manifesta una notevole difficoltà nella traduzione. Esistono, però, diverse soluzioni; gli approcci prescrittivi qui potrebbero portare a deviazioni fonologiche o lessicali e causare delle inadeguatezze. Roman Jakobson elabora delle teorie di grande valore in merito; nel suo saggio On Linguistic Aspects of Translation egli introduce concetti cruciali per gli studi di traduttologia, sostenendo che non esiste un'equivalenza completa, bensì due messaggi equivalenti con due codici differenti tra due culture diverse:

We distinguish three ways of interpreting a verbal sign: it may be translated into other signs of the same language, into another language, or into another nonverbal system of symbols. (Jakobson, 1959: 233) 
Tre sono i concetti principali introdotti dal grande esponente del formalismo russo: una traduzione intralinguistica cioè una riformulazione del segno linguistico, una traduzione interlinguistica o traduzione intersemiotica o trasmutazione. È proprio quest'ultima strategia traduttiva che segna una grande problematica dall'approccio prescrittivo ed evidenzia l'impossibilità di un'equivalenza completa fra i sistemi culturale e linguistico del testo di partenza e quelli del testo di arrivo.

A partire dagli anni Settanta la tendenza generale negli studi di traduzione sposta l'enfasi sulla traduzione come prodotto finale, come nelle teorie funzionaliste della scuola tedesca i cui esponenti sono Hans J. Vermeer e Katharina Reiß che concepiscono la traduzione come atto di comunicazione interculturale (Munday, 2012: 133). Reiß si interessa alla traduzione della letteratura per l'infanzia e si concentra nel delineare gli aspetti che distinguono la traduzione per l'infanzia da qualsiasi altra traduzione. Il primo aspetto riguarda l'asimmetria comunicativa nel senso che il risultato del processo traduttivo è determinato dalle supposizioni che il traduttore riesce a formulare; queste supposizioni devono essere assimilate dal pubblico infantile e «adeguate» alla cultura d'arrivo, magari modificando il testo in base a principi pedagogici o educativi e prendendo in considerazione le conoscenze limitate del mondo dei bambini a quest'età (Pederzoli, 2012: 60). Nel nostro corso, queste teorie vengono spiegate dal docente e discusse a lungo con gli studenti che hanno adottato diverse strategie nella traduzione dei giochi di parole in Favole al telefono. Ecco gli esempi della prima strategia:

Tabella 7. Gioco di parole

1. È uno «stemperino», cioè un temperino con l'esse davanti. Serve a far ricrescere le matite, quando sono consumate, ed è molto utile nelle scuole.

(Rodari, FT: 25)

2. Poi abbiamo lo «staccapanni».

- Vorrà dire l'attaccapanni.

-L'attaccapanni serve a ben poco, se non avete il cappotto da attaccarci. Col nostro «staccapanni» è tutto diverso. Li non bisogna attaccarci niente, c'è già tutto attaccato. Se avete bisogno di un cappotto andate li e lo staccate. Chi ha bisogno di una giacca, non deve mica andare a comprarla: passa dallo staccapanni e la stacca. C'è lo staccapanni d'estate e quello d'inverno, quello per nomo e quello per signora. Cosi si risparmiano tanti soldi.

(Rodari, FT: 25)

3. Poi abbiamo la macchina «sfotografica», che invece di fare le fotografie fa le caricature, così si ride.

(Rodari, FT: 26)

4. Lo «scannone» è il contrario del cannone, e serve per disfare la guerra. [...] può adoperarlo anche un bambino. Se c'è la guerra, suoniamo la stromba, spariamo lo scannone e la guerra è subito disfatta.

(Rodari, FT: 26)
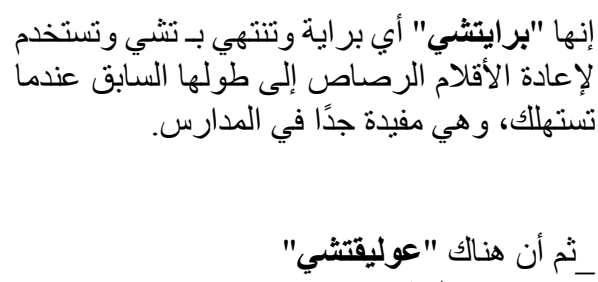

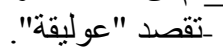

ـالعوليقة تستخدم في القليل من الأغراض، فقط

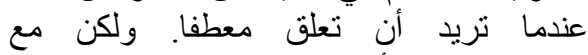

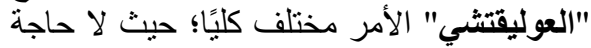

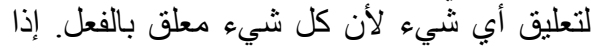

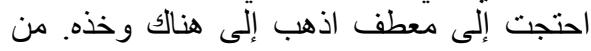

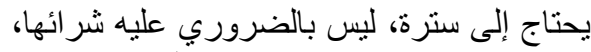

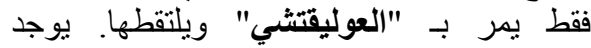

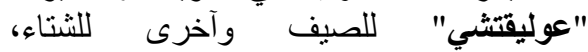
"عوليقتشي" للرجال وآخرى للنساء، هكذا نوفر للنئ

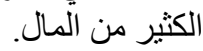

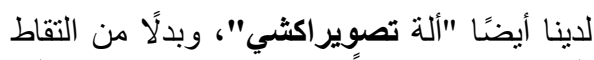
الصور ترسم صور أ كاريكاتيرية، و هكذا نضحك.

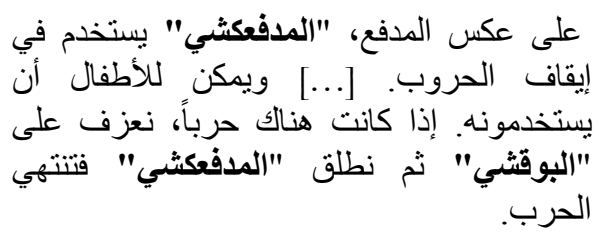


Qui il gioco di parole si basa sulla creazione di parole non lessicalizzate, cioè che non entrano nell'uso comune. Nella maggior parte degli esempi, lo scrittore dipende dall'aggiunta di un prefisso alla parola per darle nuovo significato; "stemperino", "staccapanni", "sfotografica", "scannone" e "stromba"; la $s$ di valenza negativa viene tradotta

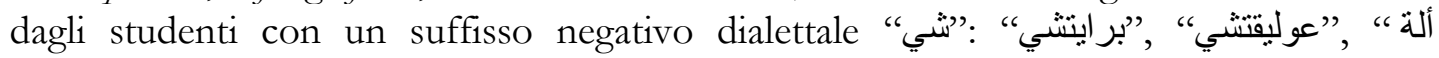
"تصوير اكثي " البوقثي" " una traduzione molto vicina al mondo infantile, lontana dall'arabo aulico. Gli studenti sono riusciti ad accostarsi il più perfettamente possibile al testo italiano.

Tra i più semplici giochi di parole spontanei, in cui vengono inventati dei termini o delle locuzioni pertinenti al linguaggio infantile, segnaliamo alcuni esempi in questa tabella:

Tabella 8. Gioco di parole

1. Allora inventiamo in fretta altri numeri per finire. $L i$ dico io, alla maniera di Modena: unci dunci trinci, quara quarinci, miri miminci. un fan dès. -E io li dico alla maniera di Roma: unzi donzi trenzi, quale qualinzi, mele melinzi. riffe raffe e dieci.

(Rodari, FT: 32)

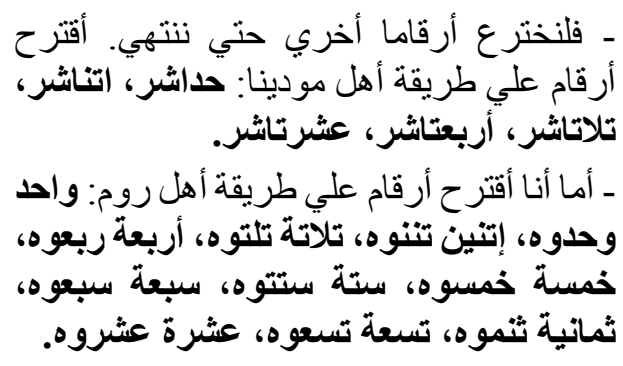

2. La domestcia fece il conto [...]: ci volevano tremendamila, tredici tredicioni e mezzo

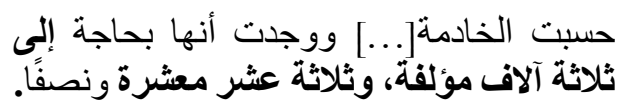
(Rodari, FT, 61)

3. Non si dice "la luna" ma "Iun lun"

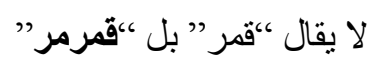

(Rodari, FT: 138)

4. La polenta" si dice "Iun polentun"

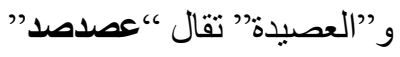

(Rodari, FT: 138)

Nei numeri inventati "unei dunci trinci, quara quarinci", "un₹i donæi trenæi, quale qualin₹?", "tremendamila, tredici tredicion?" dove lo scrittore conserva una parte della parola aggiungendo altre desinenze che formano tutte insieme una rima, gli studenti riescono ad adattare il gioco all'arabo mantenendo la rima e rimanendo fedeli allo stesso campo semantico, quello numerico. Anche quando lo scrittore va avanti con il gioco inventando parole completamente non lessicalizzate che mantengono l'effetto sonoro "miri miminci. un fan dès", "mele melinzi. riffe raffe", gli studenti inventano dei numeri che mantengono la

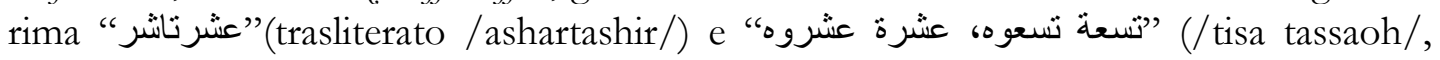
/ashra asharoh/).

Negli esempi "lun lun" e "lun polentun" Rodari parla di un luogo immaginario in cui tutti i sostantivi iniziano con "lun", che forma una specie di prefisso, un procedimento fattibile in una lingua musicale come l'italiano. Traducendo, gli studenti non hanno potuto a trovare delle modulazioni lessicalizzate equivalenti e optano per ripetere l'ultima sillaba della parola araba: " compensando lo stile e il contenuto semantico e cambiando il gioco linguistico con un altro.

Tradurre il gioco di parole del testo originale con un gioco nella lingua di arrivo è una delle strategie di cui parla Delabastita (1996) che postula la traducibilità del gioco di parole, quanto meno in termini funzionali. A tal fine sono possibili diverse strategie. A volte il 
(C) Italiano LinguaDue 2. 2021. Samah Mohammed Ibrahim Abdo, Problematiche di tradurione letteraria dallitaliano in arabo in un modello didattico applicato

pun può essere riprodotto quasi identico, a volte può essere sostituito con un altro, soprattutto quando il gioco fa leva su una figura retorica (come la ripetizione, l'allitterazione, la rima, l'ironia, il paradosso, ecc.). Si leggano i seguenti esempi:

Tabella 9. Altri giochi di parole

1. tre per uno Trento e Belluno tre per due bistecca di bue tre per tre latte e caffè tre per quattro cioccolato tre per cinque malelingue tre per sei patrizi e plebei tre per sette torta a fette tre per otto piselli e risotto tre per nove scarpe nuove tre per dieci pasta e ceci

(Rodari, FT: 31)

2. Una buffa filastrocca nella lingua del pianeta Beb che diceva:

\section{anta anta pero pero penta pinta pim però e io non ci ho capito niente.}

(Rodari, FT: 144)

3. Brif, brof, braf, - disse il primo.

Braf, brof, - rispose il secondo. E scopiarono a ridere.

(Rodari, FT: 33)

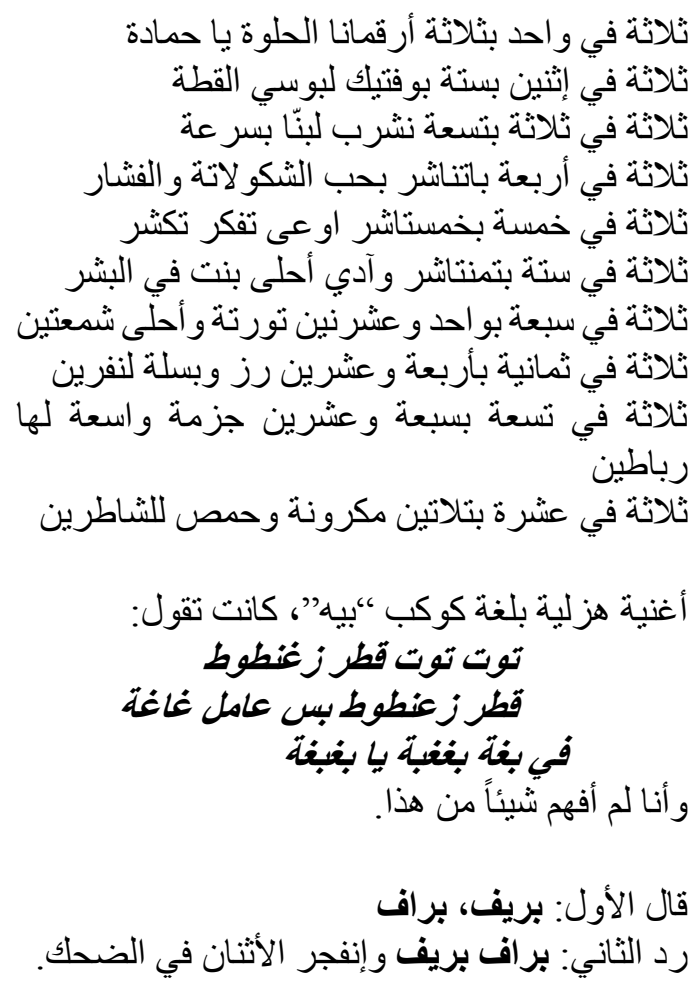

ثلاثة في واحد بثلاثة أرقمانا الحلوة با حمادة

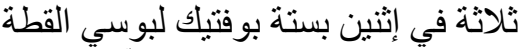

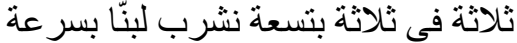





ثلاثة في ستة بتمنتانشر و آدي أحلى بنت في في البشر تئر

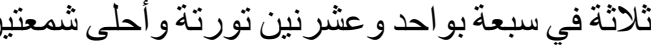

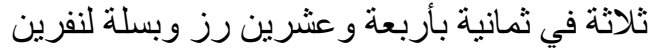



رباطين

ثلاثة في

أغنية هزلية بلغة كوكب “بيه”، كانت تقول.

توت توت قطر زغنطوط

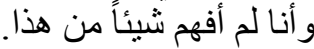

قال الأول: بريف، براف بريف

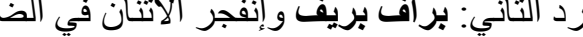

Nel primo esempio gli studenti hanno scelto di sostituire il gioco di parole con un altro gioco di parole; sono riusciti a riprodurre l'umorismo delle combinazioni inventate dallo scrittore con una specie di filastrocca inventata da loro stessi, pur rimanendo fedeli agli

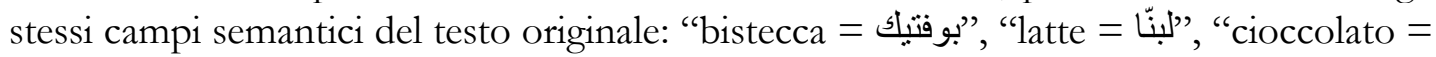


=زمة", "مكرونة وحمص ="pasta e ceci", inoltre hanno mantenuto la rima e l'effetto sonorio sempre piacevole ai bambini.

Nel secondo esempio hanno scelto di avvicinare il testo italiano alla cultura di arrivo, traducendo i due versi "anta anta pero pero/ penta pinta pim perö" con due versi di una canzone egiziana per i bambini, già conosciuta " "توت توت " ricca di parole inventate e di assonanza anch'essa.

Con la loro sensibilità artistica e letteraria, gli studenti affrontano quel rischio che è stato definito della «supertraduzione» (Mounin, 1965: 148), cioè la condizione in cui il traduttore si sovrappone all'autore adattando lo stile e $\mathrm{i}$ valori del testo originale alla lingua di arrivo. Tuttavia esiste anche la possibilità che il testo tradotto acquisti elementi formali o semantici che non sono espressi nell'originale, che nascono dalle possibilità espressive del nuovo materiale linguistico (cfr. Mura, 1989: 422).

Nel terzo esempio i protagonisti, due bambini, usano una lingua propria, fatta di parole inventate così nessun altro li potrebbe capire. Sono delle combinazione che non hanno nessun significato ma di effetto sonoro. Nella traduzione gli studenti optano per una 
بريف “ برئ "trascrizione per mantenere il gioco fonetico grazie ad un'assonanza approssimativa بورف بر اف", riproducendo la stessa ambiguità nella lingua d'arrivo.

Fra le altre strategie di cui parla Delabastita è la traduzione del gioco di parole con una frase che non contiene un gioco, ma mantiene il significato a costo di sopprimere il gioco. Ecco gli esempi:

Tabella 10. Giochi di parole

1. ... aveva realizzato un risparmio di due stramilioni (Rodari, FT: 20)

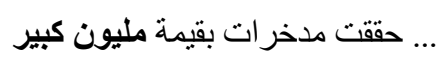

2. È troppo poco. Senti questi: uno stramilione di biliardoni. Un ottone di millantoni. Un meravigliardo e un meraviglione.

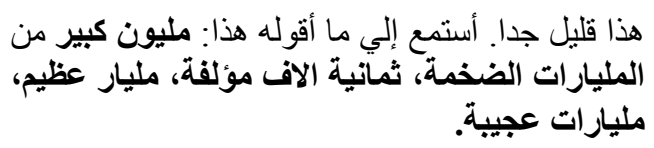

(Rodari, FT: 31)

3. Tristecca ai ferri (Rodari, FT: 141)

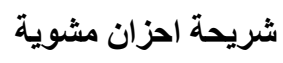

Per ottenere l'effetto di esagerazione con i numeri, lo scrittore gioca con le parole facendo leva sull'aggiunta di un suffisso (one/i: biliardoni, ottone, millantoni) o un prefisso (stra: stramilioni) o sulla fusione di due termini (meravigliardo che sta per meraviglia + miliardo, meraviglione che sta per meraviglia + milione). La traduzione ha soppresso il gioco مليون كبير من المليارات “ "

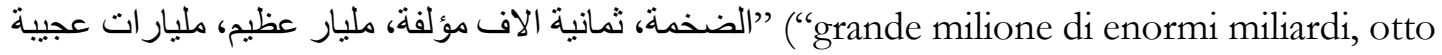
migliaia di migliaia, enorme milione, meravigliosi miliardi”).

Nel terzo esempio, "Tristecca ai ferri" è una parte di un menù immaginario: il gioco si basa sulla combinazione delle due parole "bistecca" e "tristezza". La traduzione letterale شui mantiene l'equivoco nel senso ma non l'effetto umoristico del gioco “ شريحة أحزان " مشوية" ("una bistecca di tristezza”). Agli studenti non riesce una traduzione omomorfa del meccanismo di creazione dell'equivoco, che tuttavia perde la sfumatura del gioco di parole originale. La difficoltà di questo genere è soprattutto dovuta alla differenza tra le due lingue per quanto riguarda il lessico e la struttura (Delabastita, 1996; Vandaele, 2011). La totale rinuncia al gioco di parole può risultare una scelta obbligata.

Come vediamo, molti dei giochi di parole sopra analizzati sono stati sostituiti con altri giochi di parole appartenenti alla lingua di arrivo. La riproduzione dei giochi di parole contribuisce alla conservazione dell'umorismo che caratterizza il libro originale. Abbiamo anche notato che, nonostante l'intenzione degli studenti di riprodurre la maggior parte dei giochi linguistici nel testo di arrivo, questo non è sempre stato possibile.

\section{GLI ANTROPONIMI}

Ogni lingua è caratterizzata da un sistema approvato e condiviso di nomi propri, definiti antroponimi, generalmente riconoscibili dai loro fruitori come nomi convenzionali appartenenti al sistema ordinario dei nomi (Bertills, 2003: 17).

Oltre a essere mezzi che "designano un particolare "individuo" di una specie o categoria; un essere umano (Carla), una nazione (Francia), una città (Bergamo), ecc» (Dardano, Trifone, 1997: 167), i nomi identificano un referente unico e «monoreferenziale» come afferma Christiane Nord: 
A proper name can tell us whether the referent is a female or male person (Alice - Bill), maybe even about their age (some people name their new-born child after a pop star or a character of a film that happens to be en vogue) or their geographical origin within the same language community (e.g., surnames like McPherson or O'Connor, a first name like Pat) or from another country, a pet (there are "typical" names for dogs, cats, horses, canaries, etc., like Pussy or Fury), a place (Mount Everest), etc. Such indicators may lead us astray in real life, but they can be assumed to be intentional in fiction. (Nord, 2003: 183)

Il nome può indicare informazioni di vario tipo, come le presumibili caratteristiche del personaggio o del suo aspetto fisico, la fascia di età o il contesto sociale, ecc. Considerando i personaggi come personificazioni di difetti, di virtù o di qualità relative alla vita umana, è importante comprenderne il significato semantico, poiché fornisce al lettore una certa idea, dello status sociale e del ruolo rivestito dal personaggio, e, in generale, di quello che ci si può e, in un certo qual modo, ci si deve aspettare da esso (Manini, 1996: 165, 166).

Theo Hermans (1988), Luca Manini (1996), Michel Ballard (2001), Yvonne Bertills (2003) e Maurizio Viezzi (2004) sono stati tra i primi a studiare e ad affrontare tale questione dal punto di vista traduttivo. Gli approcci traduttivi adottati variano dal mantenimento del nome straniero all'adattamento del nome alla lingua di arrivo. In generale, la strategia più utilizzata per la traduzione dei nomi propri delle opere scelte, è la prima, cioè il mantenimento del nome originale, perché sono nomi che appartengono al linguaggio quotidiano e che non dovrebbero essere alterati senza una ragione forte, come sostiene Klingberg (1986). Così la presenza di questi nomi nel testo di arrivo attira l'attenzione del lettore, ricordandolo che il testo appartiene alla sfera culturale italiana.

Data l'importanza della funzione svolta dai nomi dei personaggi, gli studiosi di letteratura per l'infanzia e i traduttori di libri per bambini sono chiamati ad affrontare degli ostacoli legati sia alla formazione e alla comprensione dei nomi sia alla loro traduzione. L'unica opera del corpus a rappresentare un vero problema per gli studenti nella traduzione dei nomi è Le avventure di Cipollino per via del forte carico semantico intrinseco dei nomi; non tradurre tali nomi avrà conseguenze sulla comprensione del testo. Infatti, oltre alla presenza di personaggi dalle chiare sembianze umane, sono molto frequenti oggetti animati (Cipollino, Limonaccio), animali (Talpa, Topolino, ecc..) con caratteristiche umane. Tale peculiare natura dei personaggi letterari influenza la formazione dei loro nomi; nello specifico, i personaggi immaginari ricevono nomi che possono contenere specifici significati, a differenza dei nomi delle persone reali che sono caratterizzati da forme più convenzionali o neutrali (cfr. Bertills, 2003: 233). Non tradurre questo tipo di nomi provocherebbe un effetto alienante sui lettori del testo tradotto (Puurtinen, 1995), e porterebbe al rischio dell'abbandono completo della lettura da parte del bambino in quanto i nomi nella lingua straniera risultano troppo difficili da leggere e da decodificare, e quindi possono influire negativamente sul processo di identificazione e di memorizzazione.

Per gli studenti il compito più arduo era trasportare lo stesso effetto semantico dal testo di partenza a quello di arrivo; ne Le avventure di Cipollino i nomi hanno delle funzioni concomitanti: divertire il lettore, creando effetti comici; caratterizzare tratti della personalità dei personaggi; trasmettere informazioni, oppure suscitare emozioni. Qui capire il significato dei nomi è particolarmente importante durante la lettura perché i nomi rivestono un ruolo fondamentale nel processo d'identificazione del lettore-bambino (Nikolajeva, 2002: x). Gli esempi seguenti mettono in evidenza le difficoltà del processo traduttivo di alcuni nomi di personaggi e le manipolazioni che i nomi hanno subito: 
(C) Italiano LinguaDue 2. 2021. Samah Mohammed Ibrahim Abdo, Problematiche di tradurione letteraria dall'italiano in arabo in un modello didattico applicato

Tabella 11. Gli antroponimi

1. Cipollone
Cipollino
Cipolletto
Cipollotto
Cipolluccio
2. Limoncini
Il Principe Limone
I Limoni di corte
3. Fagiolino
Il sor Zucchina
Signor Conte Ciliegione
Mastro Uvetta
Il cavalier Pomodoro

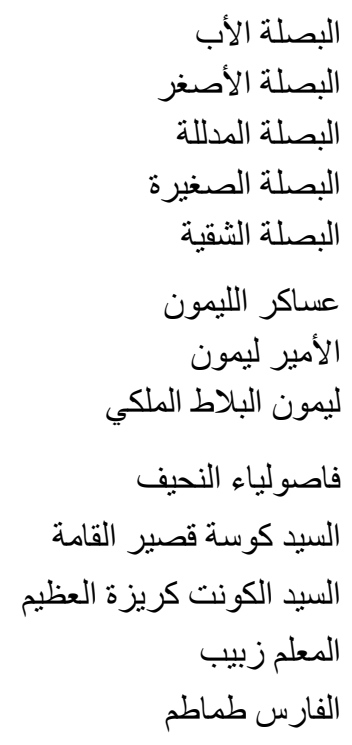

Nel processo traduttivo, gli studenti dovevano affrontare due problematiche: la prima è la traduzione del nome, la seconda è la suffissazione; la famiglia Cipollone è "gente per bene" ma "quando si nasce cipolle, le lacrime sono di casa"; capire il senso dei nomi sembra indispensabile per comprendere certi tratti del testo perché sono nomi polifunzionali in quanto trasportatori di significati semantici. Sono il padre Cipollone e i figli Cipollino, Cipolletto, Cipollotto, Cipolluccio. Il procedimento traduttivo consiste in una trasposizione per cui si sostituisce il suffisso - e l'implicazione semantica in esso contenuta - con gli aggettivi, come in: Cipollino = البصلة الأصغر ("Il Cipollo Minore"), Cipolletto = المدللة البصلة ("Il Cipollo prediletto"), Cipollotto = البصلة الصغيرة ("Il Piccolo Cipollo”), Cipolluccio = البصلة الثقية ("Il Cipollo Capriccioso"), o con nomi di parentela, come nel nome del padre "Cipollone "البصلة الأبح ("papà Cipollo"). Con questi "nomi motivati", quelli cioè che hanno una funzione semantica, questa strategia è riuscita a riprodurre l'effetto creato dall'autore.

Altri nomi che rivestono di molteplici funzioni e denotano i personaggi sono Limoncini, così acidi, in funzione della polizia, "I Limoni di corte" e "Principe Limone", il governatoretiranno: la traduzione ha sostituito i suffissi con termini che si riferiscono alla categoria professionale e riproducono le connotazioni dei nomi: “ليمون البلاط الملكي", "الأمير ليمون", "عساكر الليمون" ("Il Principe Limone", "I limoni della Corte Imperiale", "I limoni soldati”). La traduzione non implica solo la trasmissione della denotazione o il significato letterale dei nomi, ma anche la trasmissione delle loro connotazioni, ovvero il significato contestuale che cambia da testo a testo, come osserva Nikolajeva (2006: 278).

La scelta del nome di un personaggio è sempre fondamentalmente motivata, soprattutto in considerazione del suo ruolo nella narrazione, come il nome Fagiolino in cui il suffisso -ino viene tradotto con l'aggettivo "نحيف" cioè "magrolino", perché è il povero "قصير القامة" " ("basso"). Il suffisso one in "signor Conte Ciliegione" viene tradotto con l'aggettivo "العظير" ("il grande"). Sostituire i suffissi italiani con gli aggettivi in arabo sembra la soluzione più adatta perché usare questo tipo di suffissi non è comune nell'arabo attuale. In questo modo i nomi conservano i loro ruoli che rivestono nella trama dell'opera (Viezzi, 2004: $33)$. 
Con i nomi parlanti, gli studenti dovevano rendersi conto del genere del nome: "Mastro Uvetta" viene tradotto letteralmente "المعلم زبيب"; a determinarne il genere è la parola "mastro", così nell'arabo come nell'italiano. Di genere femminile in arabo è il nome "Pomodoro" che nella traduzione viene sempre anticipato da "الفارس" ("il cavaliere") per determinarne il genere. Mantenere il genere di ogni nome così com'è nella lingua originale si rivela necessario per poter mantenere le connotazioni di questi nomi e creare una familiarità nella cultura di arrivo.

Bisogna sapere che il ductus consonantico arabo non comprende le lettere maiuscole, e per scrivere un nome proprio italiano in arabo gli studenti hanno preferito usare le virgolette per rendere facilmente identificabili tali nomi, anche se in arabo gli antroponimi vengono scritti senza virgolette.

Rispettando i concetti di recognizability e memorability, introdotti da Tymoczko (1999), le strategie adottate dagli studenti per affrontare le problematiche sopra indicate, hanno l'obiettivo di facilitare, in qualche misura, la lettura del testo tradotto ai bambini con l'adattamento dei nomi dei personaggi alla cultura di destinazione. Così si evitano i problemi di comprensione del testo, nonché le sensazioni di smarrimento.

\section{LE ONOMATOPEE}

Un'altra problematica della traduzione riguarda le onomatopee molto ricorrenti nel romanzo a fumetti Il grande Belzoni mentre nelle altre opere hanno una frequenza scarsa. Le onomatopee sono una categoria di parole che ha usi e funzioni simili alle interiezioni; esse imitano suoni o rumori attraverso grafemi nello scritto o fonemi nel parlato (miao, tin tin, ₹:2), condividendo con le interiezioni l'assenza di legami sintattici con il resto della frase. Si suddividono in onomatopee semplici e derivate: le semplici sono quelle sequenze di caratteri che riproducono certi suoni o rumori (brr, bum, dindon, o ancora i versi degli animali: bau, cra cra), mentre le derivate, dette anche "parole onomatopeiche" (come: miagolare, tintinnio, ronzare, cinguettio, ticchettio, brontolare, mormorare, sibilo e sibilare, ululo e ululare) stabiliscono legami sintattici con il resto della frase (Dardano, Trifone, 1983: 440).

Nei fumetti italiani, le onomatopee non si sono liberate dell'influenza inglese nonostante i tentativi di «italianizzazione» e di adattamento alla fonetica italiana; grazie alla diffusione dei fumetti americani, le onomatopee sono diventate comprensibili a livello universale: «In tutte le lingue molte parole si sono formate per somiglianza con il suono prodotto dalla cosa che dovevano indicare è il caso delle onomatopee o parole fonosimboliche»s (Garajová, 2014: 75). Ecco alcuni esempi dei numerosi prestiti onomatopeici dall'ambito anglosassone nel romanzo a fumetti Il grande Belzoni:

Tabella 12. Le onomatopee

\begin{tabular}{|c|c|c|}
\hline 1. & BANG & طاخ \\
\hline 2. & BLUB & بلوب بلوب \\
\hline 3. & BLUGK & بلج بلج \\
\hline 4. & CRAK & كر الك \\
\hline 5. & CRASH & تحطم \\
\hline 6. & KRAK & اصطدام \\
\hline 10 & SKRAK & سكر الك \\
\hline & SKRSH & سكر اش \\
\hline
\end{tabular}


(C) Italiano LinguaDue 2. 2021. Samah Mohammed Ibrahim Abdo, Problematiche di tradurione letteraria dall'italiano in arabo in un modello didattico applicato

\begin{tabular}{|c|c|}
\hline 9. SOCK & لكمة \\
\hline 10. SPLASH & طش \\
\hline 11. SWISH & سويش س \\
\hline 12. TOC TOC & تالك تالك \\
\hline 13. TUMP & ارتطام \\
\hline 14. WAMP & اشتعال \\
\hline
\end{tabular}

Lo scrittore opta per onomatopee anglofone per rendere più dinamica la rappresentazione della storia, per cui lo sparo diventa "BANG", il suono del fucile diventa "CRAK", "CRASH" per il suono di scontro, "SOCK" per il colpo di pugno, "SPLASH" per il rumore dell'acqua, "SWISH" per il fruscio, "WAMP" per il fuoco che subito divampa, e così via. Qui l'italiano ha assunto la rappresentazione lessicale dall'inglese, e ciò finisce per integrare nella traduzione un prestito di secondo grado come "كرالك" e

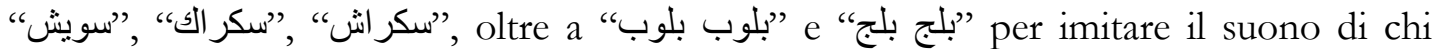
annega. Le onomatopee riportano le caratteristiche peculiari dell'oralità, come in "BANG

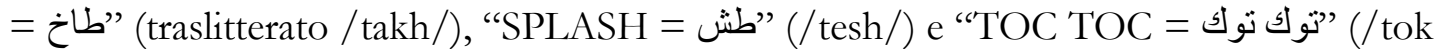
tok/).

Tuttavia, in tanti esempi gli studenti sono riusciti a trovare delle onomatopee derivate

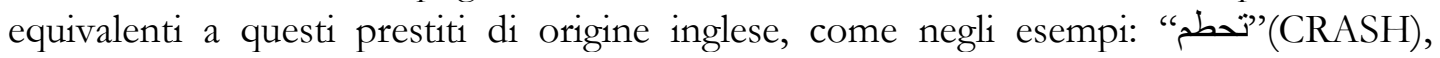

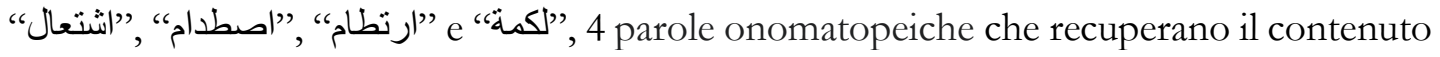
semantico delle onomatopee del testo originale.

La linguistica contrastiva spiega come ogni lingua può avere diversamente le proprie onomatopee; per lo stesso referente cambia la rappresentazione lessicale nelle diverse lingue, come negli esempi:

Tabella 13. Le onomatopee
1. AAGKH
2. BDUM
خالخ خالخ
3. $\mathrm{COFF}$
4. RRUMMBLEE, BDOOM
5. STUD
6. STUM
7. SWISS, FRECCIA, ARCO

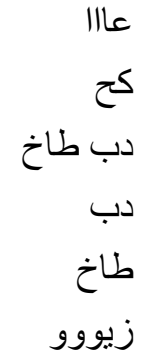

Queste onomatopee vengono adattate alla fonologia e alla morfologia araba. Il suono di tosse umano è lo stesso ovunque, ma in italiano si sente e si rappresenta con COFF mentre in arabo si fa "كح" (traslitterato / koh/), "SWISS" per il suono della freccia diventa "خالخ خالخ "(/ziwww/), “AAGKH” di un moribondo diventa " (/khaakh/khaakh/, "BDUM" per il verso dello iena attaccante diventa "عالاخ" (/'aah/), "RRUMMBLEE, BDOOM" per il tuono e la folata di vento diventa "دب "طاخ " (/dip/taakh/), e così via. Qui il lessico utilizzato nella lingua d'arrivo è quello della quotidianità e rispecchia un registro informale e non-sorvegliato. Occore precisare che in arabo le vocali delle onomatopee sono necessariamente lunghe, perché nella lingua araba le vocali brevi non sono delle lettere, ma dei segni facoltativi che vengono collocati sopra del ductus consonantico: vengono segnati semplicemente con un trattino sopra la lettera 
per indicare una breve $a$, un altro sotto per indicare una breve $i$. Analoghe considerazioni si possono avanzare per il timbro vocalico: le vocali e, o della lingua sorgente debbono per forza essere rese, e si neutralizzano, con le vocali i, u (lunghe).

A volte gli studenti hanno sentito la necessità di leggere qualche fumetto scritto in arabo, come la versione araba di Topolino, per vedere come si traspongono certi suoni. Le onomatopee devono rapportarsi al contesto visivo per poter trasmettere una forza comunicativa grafica e verbale: un «booOM», dove le lettere diventano via via sempre più grandi e visibili significa, non solo «un'esplosione» ${ }^{8}$, ma «un'esplosione apocalittica» come c'è un'enorme differenza tra un semplice «bum», un «BUM» scritto in maiuscole. Ne $I l$ grande Belzoni le onomatopee forgiate appositamente per imitare vari suoni, appaiono in grassetto e sempre in maiuscole e diventano un elemento grafico che entra in relazione con lo sfondo, oppure inscritte nella vignetta. Si notino questi due esempi:

\section{SWIIISH سوييش, \\ دوم دووم دوم دووم DUMS DUMS DUMS DUMS}

Gli esempi dimostrano in modo molto conciso - per adattarsi alla vignetta - non solo un suono, ma anche la sua durata - una lunga durata grazie alla ripetizione della $i$ nel primo esempio - nonché la sua forza come nel secondo esempio. Lo scopo degli studenti è quello di portare il testo dalla lingua d'origine alla lingua di destinazione in maniera tale da mantenere inalterato il più possibile il significato e lo stile del testo originale.

\section{CONCLUSIONE}

Nella traduzione di testi letterari dallitaliano in arabo, le problematiche lessicali dei nostri studenti sono tra le più frequenti e sono molto diverse tra loro. Il nostro obiettivo è quello di presentarne i più sistematici. La ricerca mira ad aiutare gli studenti a migliorare le loro abilità ricettive di un testo letterario, di motivarli ad avvicinarsi alla letteratura italiana e alla lettura di altre opere dello stesso genere letterario in italiano o tradotti, oltre a sviluppare un certo gusto per la cultura e l'etimologia e la ricerca di somiglianze e differenze lessicali e strutturali tra italiano come LS e l'arabo come L1.

Nella nostra analisi, ci siamo accorti che la traduzione delle espressioni idiomatiche rappresenta una delle difficoltà maggiori; una prima osservazione generale è che, a volte, i dizionari non costituiscono un valido aiuto per gli studenti: l'ambiguità tra senso letterale e senso figurato da una parte, e l'opacità del messaggio dall'altra parte, portano a una traduzione letterale non adeguata.

Oltre alle lacune che i dizionari monolingue a volte presentano, la maggior parte dei dizionari bilingui italiano-arabo, non raccoglie le espressioni idiomatiche.

L'analisi delle traduzioni ha dimostrato che le parole/espressioni polisemiche rappresentano una sfida particolare; per orientarsi, lo studente deve cercare spunti nel contesto per scegliere il significato adatto. Qui non basta consultare il dizionario. Optare per una traduzione inappropriata di un termine polisemico, potrebbe essere dovuto all'equivoco tra la lingua madre e l'italiano come LS, o a una traduzione affrettata in cui lo studente sceglie la parola in un significato che conosce già, senza consultare il dizionario per individuare altri significati più adeguati.

${ }^{8}$ Eco (2008): https://espresso.repubblica.it/opinioni/la-bustina-di-minerva/2008/11/28/news/mumblemumble-crash-1.11062/). 
Tra le 7 opere del nostro corpus, almeno sono 3 le opere scritte per bambini: Favole al telefono, Le avventure di Cipollino, Fiabe Italiane, perciò mi sono focalizzata su elementi della letteratura d'infanzia di particolare difficoltà nella traduzione, quali i giochi di parole e la traduzione degli antroponimi.

Con i giochi di parole gli studenti riescono a formulare delle supposizioni assimilate dal pubblico infantile e adeguate alla cultura d'arrivo. A volte si riesce a riprodurre identico il gioco, a volte esso viene sostituito con un altro, soprattutto quando il gioco fa leva su una figura retorica. Sono delle «supertraduzioni»: il traduttore si sovrappone all'autore adattando lo stile e i valori che sono propri della lingua di arrivo. Tuttavia esiste anche la possibilità che il testo tradotto acquisti elementi formali o semantici che non sono espressi nell'originale.

Con le parole inventate diventa necessario cercare di creare un neologismo soprattutto quando si tratta di un lemma il cui sfondo culturale non ha un corrispondente nella lingua d'arrivo. A differenza della grammatica, il lessico presenta un sistema aperto in continuo cambiamento e la creatività lessicale risulta una sorgente d'arricchimento di ogni lingua.

Tuttavia quando non è possibile tradurre il gioco di parole nella lingua d'arrivo (a causa della differenza tra le due lingue per quanto riguarda il lessico e la struttura), si può optare per il mantenimento del significato a costo di sopprimere il gioco. La totale rinuncia al gioco di parole può risultare una scelta obbligata.

$\mathrm{Ne}$ Le avventure di Cipollino gli studenti hanno dovuto affrontare il problema della traduzione degli antroponimi trasportando lo stesso effetto semantico dal sistema di partenza a quello di arrivo. Capire il significato dei nomi e mantenere le loro connotazioni è particolarmente importante durante la lettura perché i nomi rivestono un ruolo fondamentale nel processo d'identificazione del lettore-bambino.

Le strategie adottate dagli studenti nella traduzione dei nomi hanno l'obiettivo di facilitare, in qualche misura, la lettura del testo tradotto ai bambini nella cultura di destinazione. Così si evitano i problemi di errata comprensione, nonché sensazioni di smarrimento o, peggio, l'abbandono completo della lettura da parte del bambino in quanto i nomi nella lingua straniera risultano troppo difficili da decodificare.

L'intento di questa sezione dedicata agli antroponimi è, dunque, stabilire se la traduzione dei nomi abbia portato a un arricchimento oppure a una perdita, a livello semantico o connotativo, rispetto al testo originale e se ciò abbia inciso, in definitiva, sulla resa finale dell'opera tradotta.

Il grande Belzoni, una delle 7 opere tradotte, presenta il problema della traduzione delle onomatopee. In alcuni casi gli studenti si sono sentiti liberi di tradurre le onomatopee con onomatopee della L1 equivalenti (dato che ogni lingua può avere proprie onomatopee; per lo stesso referente cambia la rappresentazione lessicale nelle diverse lingue) o di ricorrere ad un prestito attingendo a forme onomatopeiche di origine inglese soprattutto quando mancano le equivalenti tra italiano e arabo.

Con questo corso, molto vicino al un laboratorio di traduzione, gli studenti si sono lanciati in territori mai esplorati, sperimentano strategie nuove in grado di colpire un pubblico giovanile, ricettivo e pieno d'immaginazione.

Dopo aver gettato luce sulle sfide e sulle soluzioni possibili, spero che la presente ricerca possa essere di aiuto a coloro che insegnano l'italiano a studenti arabofoni che hanno già raggiunto un certo livello di competenza linguistica. 
(C) Italiano LinguaDue 2. 2021. Samah Mohammed Ibrahim Abdo, Problematiche di tradurione letteraria dall'italiano in arabo in un modello didattico applicato

\section{RIFERIMENTI BIBLIOGRAFICI}

Alliaj G. (2018/2019), Espressioni idiomatiche e interpretazione simultanea: analisi delle strategie adottate dagli interpreti del Parlamento europeo, tesi di laurea, Alma Mater Studiorum Università, Bologna:

https://amslaurea.unibo.it/18380/1/GRETA_ALLIAJ_TESI.pdf.

Bertills Y. (2003), Beyond identification, Proper names in children's literature, Åbo akademis University Press, Turku.

Casadei F. (1995), "Per una definizione di "espressione idiomatica" e una tipologia dell'idiomatico in italiano", in Lingua e Stile, 2, pp. 335-358.

Casadei F. (1996), Metafore ed espressioni idiomatiche. Uno studio semantico sull'italiano, Bulzoni editore, Roma.

Dardano M. $\left(2017^{2}\right)$, Nuovo manualetto di linguistica italiana, Zanichelli, Bologna.

Dardano M., Trifone P. ([1983] 1995 $\left.{ }^{14}\right)$, Grammatica italiana. Con nozioni di linguistica, Zanichelli, Bologna.

Dardano M., Trifone P. (1997), La nuova grammatica della lingua italiana, Zanichelli, Milano.

Delabastita D. (1996), "Wordplay and translation; dedicated to the memory of André Lefevere (1945-1996)", Vol. 2, 2, The translator, studies in intercultural communication, St. Jerome Publishing, Manchester.

Garajová K. (2014), Manualetto di stilistica italiana, Masarykova univerzita, Brno.

Jakobson R. (1959), “On Linguistic Aspects of Translation”, in Id., On Translation, edited by Reuben Arthur Brower, Harvard U Press, Cambridge, pp. 232-239.

Klingberg G. (1986), Children's Literature in the Hands of the Translators, CWK, Gleerup, Lund.

Langlotz A. (2006), Idiomatic Creativity: A cognitive-linguistic model of idiom-representation and idiom-variation in English, John Benjamins, Amsterdam-Philadelphia.

Manini L. (1996), "Meaningful Literary Names. Their Forms and Functions, and their Translations", in The Translator, 2, 2, pp. 161-178.

Mounin G. (1965), Teoria e storia della tradurione, Einaudi, Torino.

Munday J. (2012), Introducing Translation Studies. Theories and Applications, Routledge, London-New York.

Mura A. (1989), "Sulla traduzione poetica: Halas a tempo di Ripellino. Funzioni ed equivalenze degli elementi prosodici", in Europa Orientalis, pp. 395-424.

Nida E. (1964), Towards a Science of Translating. With Special Reference to Principles and Procedures Involved in Bible Translating, Leiden E. J. Brill, Boston.

Nikolajeva M. (2002), The rhetoric of character in children's literature, Scarecrow press, Lanham.

Nikolajeva M. (2006), "What do we translate when we translate children's literature?", in Beckett S., Nikolajeva M. (eds.), Beyond Baber: the European tradition in children's literature, Scarecrow Press, Lanham-Toronto-Oxford, pp. 277-297.

Nord C. (2003), "Proper names in translation for children: Alice in Wonderland as a case in point", in Meta 48, 1-2, pp. 182-196.

Pederzoli R. (2012), La traduction de la literature d'enfance et de jeunesse et le dilemme du destinataire, Peter Lang, Bruxelles.

Puurtinen T. (1995), Linguistic acceptability in translated children's literature, University of Joensuu, Joensuu.

Redfern W. (1984), Puns, Basil Blackwell, Oxford.

Slapek D. (2016), Lessicografia computazionale e tradurione automatica. Costruire un dirionariomacchina, Franco Cesati Editore, Firenze.

Tymoczko M. (1999), Translation in a postcolonial context, St Jerome, Manchester.

Ulrych M. (1992), Translating Text: from Theory to Practice, CIDEB, Rapallo. 
(C) Italiano LinguaDue 2. 2021. Samah Mohammed Ibrahim Abdo, Problematiche di tradurione letteraria dall'italiano in arabo in un modello didattico applicato

Vandaele J. (2011), Wordplay in translation, Benjamins, Amsterdam.

Viezzi M. (2004), Denominaz̧ioni proprie e traduzione, LED, Milano.

\section{Sitografia}

Eco U. (2020), Mumble mumble crash, 28 novembre 2008:

https://espresso.repubblica.it/opinioni/la-bustina-di-

minerva/2008/11/28/news/mumble-mumble-crash-1.11062/)

https://www.almaany.com/

https://www.treccani.it/

\section{Dizionari}

Grande Dizionario Garzanti di Italiano, (2006), Editore Garzanti Linguistica, Milano.

Tellisi K. M. (1986), Diæionario italiano arabo, Addar Al Arabia Lil Kitab, Libraire Du Liban. 